\title{
GEMs With Double Layered Micropattern Electrodes and Their Applications
}

\author{
Antonio Di Mauro, Paolo Martinengo, Eugenio Nappi, R. Oliveira, V. Peskov, F. Pietropaolo, and P. Picchi
}

\begin{abstract}
We have developed and tested several new designs of GEM detectors with micropattern electrodes manufactured by microelectronic technology. In one design, the inner layer of the detector's electrode consists of thin metallic strips and the outer layer is made of a resistive grid manufactured by a screen printing technology. In other designs, the electrodes were made of metallic strips fed by HV via micro-resistors manufactured by a screen printing technology. Due to these features, the new detectors have several important advantages over conventional GEMs or ordinary thick GEMs. For example, the resistive grid (in the first design) and the screen printed resistors (in other designs) limited the current in case of discharges thus making these detectors intrinsically sparkprotected.

We will here describe our tests with the photosensitive versions of these detectors (coated with CsI layers) and the efforts of implementing them in several applications. In particular, we will focus on our activity towards the ALICE RICH detector upgrade and on tests of simplified prototypes of cryogenic dark matter detectors.
\end{abstract}

Index Terms-Cryogenics, GEM detectors, micropattern gas chambers, RICH detectors.

\section{INTRODUCTION}

$\mathbf{H}$ OLE-TYPE gaseous multipliers [1]-[4] due to their unique properties offer new possibilities in the detection of photons and charged particles. The most commonly used hole-type detector today is the so-called Gas Electron Multiplier (GEM) [3]. It is accepted in several large-scale experiments at CERN and elsewhere. However, in spite of its great success, the GEM as any other micropattern detector, is a rather fragile device and can be easily damaged by sparks developing at high gains of operation.

The origin of these breakdowns is today well understood. In the case of the poor quality detectors, the discharges are triggered by the presence of microdefects: sharp edges, micro-particles remaining after the production both inside and outside the

Manuscript received January 05, 2009; revised March 11, 2009. Current version published June 17, 2009.

A. Di Mauro and P. Martinengo are with the PH Division, CERN, 1211 Geneva 23, Switzerland (e-mail: antonio.di.mauro@cern.ch; paolo. martinengo@cern.ch).

E. Nappi is with INFN-Bari, 70126 Bari, Italy (e-mail: Eugenio.Nappi@ ba. infn.it).

R. Oliveira is with the TS Division, CERN, 1211 Geneva 23, Switzerland (e-mail: Rui.de.Oliveira@cern.ch).

V. Peskov is with the Institute for Nuclear Research, University of Mexico, Mexico City, Mexico, and also with the PH Division, CERN, 1211 Geneva 23, Switzerland (e-mail: vladimir.peskov@ cern.ch).

F. Pietropaolo is with INFN-Padova, 35131 Padova, Italy (e-mail: Francesco. Pietropaolo@cern.ch).

P. Picchi is with INFN-Frascati, 00044 Frascati, Italy (e-mail: Pio. Picchi@cern.ch).

Digital Object Identifier 10.1109/TNS.2009.2020864 holes, dirty spots (which are often semiconductive) and so on. In good quality detectors, the breakdowns appear when the total charge in the avalanche reaches some critical value:

$$
\mathrm{Q}_{\text {crit }}=\mathrm{A}_{\max } \mathrm{n}_{0} \sim 10^{6}-10^{7} \text { electrons }
$$

where $A_{\max }$ is the maximum achievable gas gain and $n_{0}$ is the number of primary electrons created by the radiation in the active gas volume of the detector. Note that a similar limit was empirically established quite a long time ago by $\mathrm{H}$. Raether [5] for parallel-plate avalanche chambers and is respectively called the "Raether limit". However, it was recently discovered [6] that a modified Raether limit applies for every micropattern detectors: GEMs, MICROMEGAS and others. Thus in the case of the detection of single electrons $\left(n_{0}=1\right)$ the $A_{\max }$ can be as high as $10^{6}$. However, in the case of the detection of radiations producing $\mathrm{n}_{0} \gg 1$ primary electrons the maximum achievable gain will be reduced. For example, in the case of the detection of x-rays form a ${ }^{55} \mathrm{Fe}$ radioactive source (each photon creates $\mathrm{n}_{0} \sim 220$ electrons), the maximum achievable gain will be $\sim 10^{4}$ and in the case of alpha particles $\left(\mathrm{n}_{0} \sim 10^{5}\right.$ electrons $)$ the maximum sustainable gains will be below $\sim 10^{2}$.

Hence, if one uses GEMs at gains of $\sim 10^{6}$ for the detection of single photoelectrons, any radioactive background creating $\mathrm{n}_{0}>1$ primary electrons will trigger breakdowns. Therefore unfortunately, sparks are unavoidable at high gain operations. Of course, the GEM community learns how to cope with the sparking problems: they use segmented GEM (to reduce the detector's capacitance), several GEMs operating in cascade (due to the diffusion effect [7] the value of the $\mathrm{Q}_{\text {crit }}$ increases) and spark-protected electronics. However, the experience in running the GEM-based PHENIX Hadron blind detector indicates that in spite all efforts the GEMs can still be damaged by sparks [8]. This is why we recently suggested a different approach: spark-proof GEMs with resistive electrodes instead of traditional metallic ones, which we called RETGEM [9], [10]. At low gas gain and low counting rates this detector operate as a usual GEM, however in the case of high gain operations, high counting rates or sparking this detector is more resembling RPCs, for example it is intrinsically spark protected.

We recently introduced a new advanced design of the RETGEM, which combines two approaches: a spark protecting resistive layer and the high segmentation of electrodes allowing one to reduce the capacitance contributing to the discharge power [11]. In this work we present results of comparative studies of several versions of such detectors with the main focus on their possible applications as single electron detectors. In particular we were interested in investigating the feasibility 


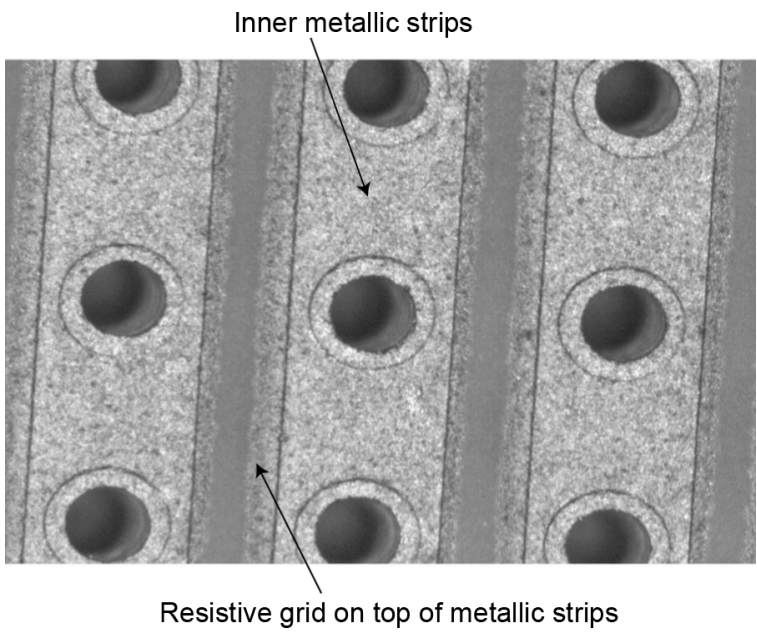

Fig. 1. A magnified photography of the S-RETGEM with holes of $0.3 \mathrm{~mm}$ in diameter. The resistive grid and the inner metallic strips are clearly visible.

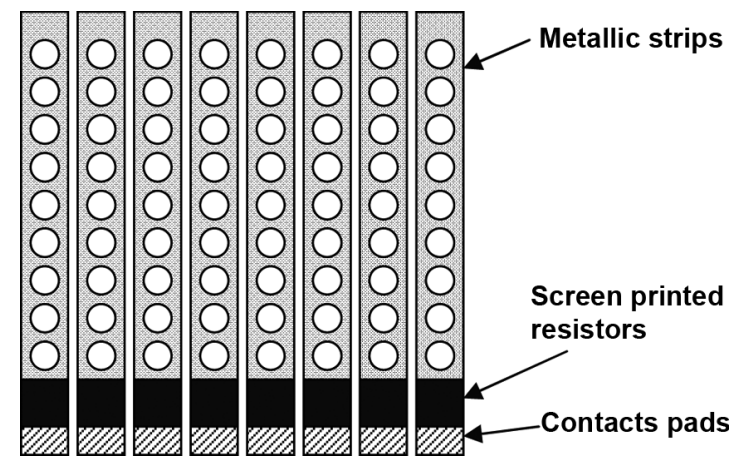

Fig. 2. A schematic drawing of the S-THGEM in which each metallic strip contains one row of holes. Strips are fed with HV via micro-resistors manufactured using a screen printing technology.

of their application to RICH and dark matter noble liquid detectors requiring single or a few electrons per event detection.

\section{DETECTOR DESIGNS AND EXPERMENTAL SETUPS}

Three spark-protected GEMs designs were developed and studied in this work. The design of the first detector, called the S-RETGEM, is described in a recent preprint [11]. It has double-layered micropattern electrodes: an inner layer consisting of thin metallic strips and an outer layer comprised of resistive grids manufactured by a screen printing technology on the top of metallic strips. The resistive layers make the detector intrinsically spark-protected. Fig. 1 shows a magnified photography of this detector on which one can see the metallic strips with holes inside and a resistive grid on top of them. In a peripheral region of this detector metallic pads where manufactured to which amplifiers can be connected or a high voltage applied.

The second detector's design was a modified "thick GEM"THGEM (see [4], [12], [13]). In this design both electrodes consisted of parallel metallic strips each of them containing one row of holes (see Fig. 2). The strip width was $0.7 \mathrm{~mm}$, the hole's diameter was $0.5 \mathrm{~mm}$ and their pitch was of $0.8 \mathrm{~mm}$; the detector's thickness was $1 \mathrm{~mm}$ and its active area of $3 \times 3 \mathrm{~cm}^{2}$. The strips on one sides of the detector were oriented perpendicular to

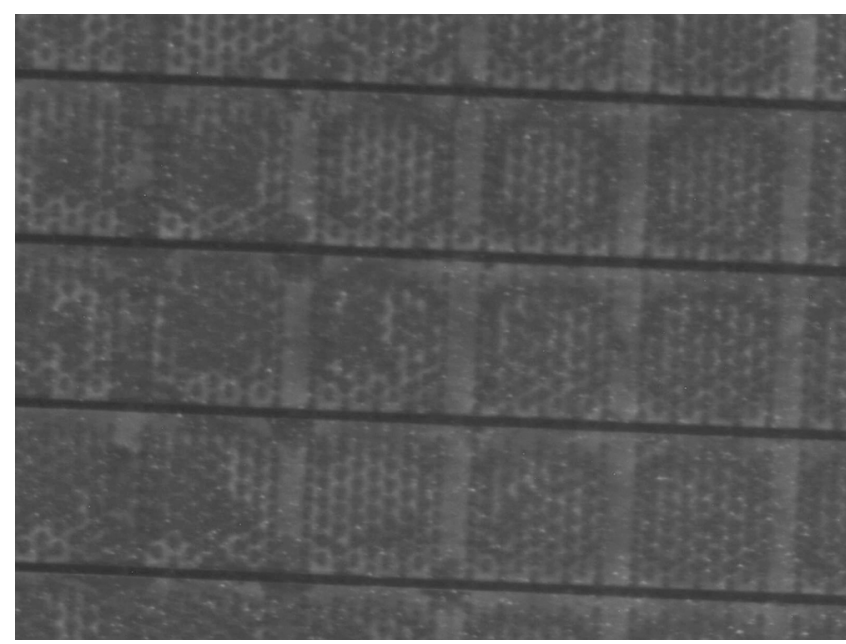

Fig. 3. A magnified photo of the S- GEM showing the separated metallic strips each containing seven rows of holes.

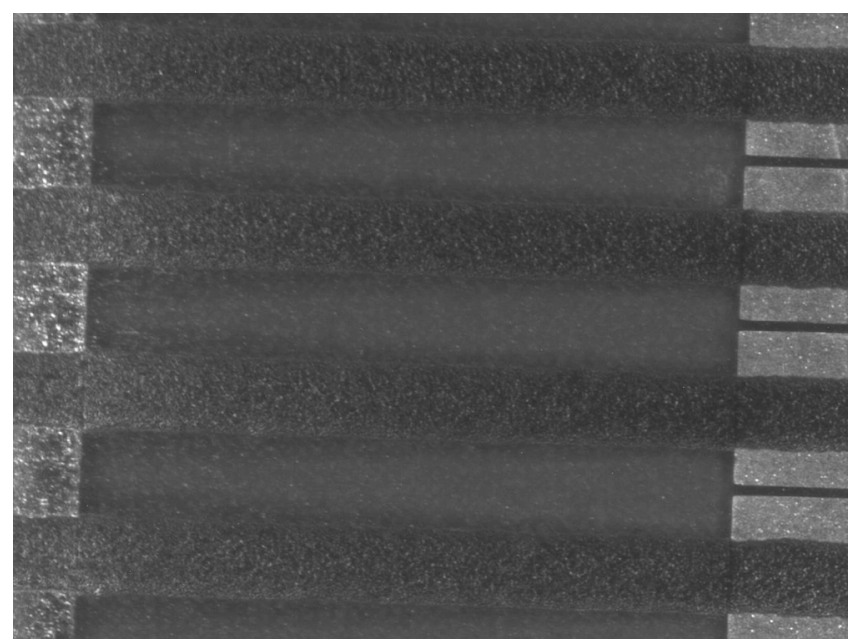

Fig. 4. A magnified photo showing micro-resistors manufactured between the metallic strips (on the right) and a high voltage electrode (on the left).

those located on the opposite side. Each strip was fed by the HV via separate micro-resistors manufactured by a screen printing technology. Such a design (we called it a "strip THGEM" or S-THGEM) even if the strips were not coated with the resistive layers, allows to considerably reduce the sparking energy (due to their low capacitance and resistivity); in this way occasional sparking does not damage the detector.

A similar concept was used in the third design: it has the same geometry as standard GEMs (the hole's diameter was $70 \mu \mathrm{m}$, the pitch $140 \mu \mathrm{m}$, the detector's thickness $50 \mu \mathrm{m}$ and the active area $10 \times 10 \mathrm{~cm}^{2}$ ), but with electrodes made of metallic strips manufactured by a photolithographic technology on the Kapton surface (we called it S-GEM). For simplicity each strip ( $1 \mathrm{~mm}$ in width) contained seven rows of holes (see Fig. 3). As in the previous design, each strip was connected to the HV electrode via micro-resistors manufactured by a screen printing technology (see Fig. 4). Strips on the opposite sides of the S-GEM were oriented perpendicular to each other.

In several comparative studies small $\left(2 \times 2 \mathrm{~cm}^{2}\right.$ active area) RETGEMs with resistive Kapton electrodes (similar to 


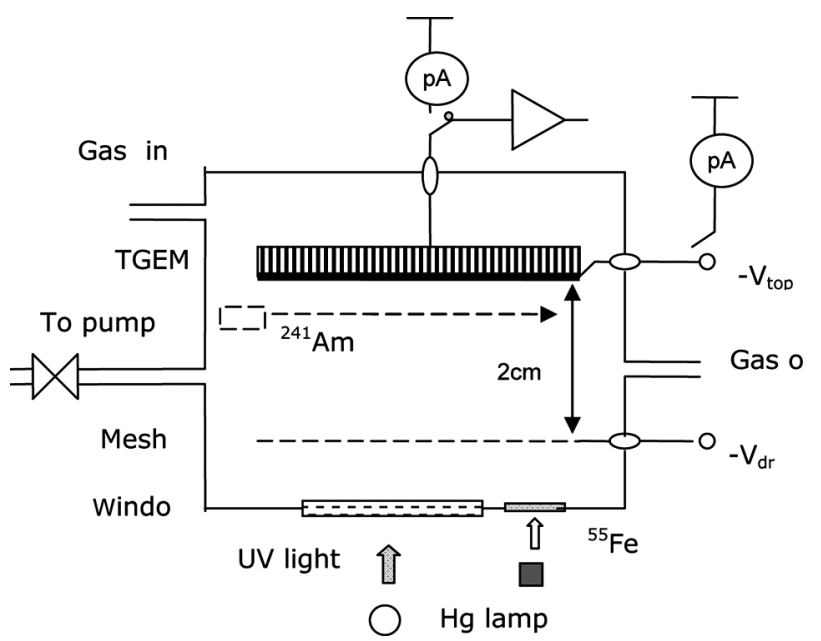

Fig. 5. A schematic drawing of the experimental setup used for the tests at room temperatures.

those described in [9]) were used. Usually such RETGEMs have higher qualities than screen printed ones allowing one to achieve almost ten times higher gas gains. One of our new Kapton RETGEM prototypes had also metallic strips manufactured in between the holes on the top of the resistive Kapton electrodes.

Two experimental setups were used in this work. One was dedicated to the study of the strip hole-type detectors for RICH applications whereas the second one was used to study their operation at cryogenic temperatures.

The schematic drawing of the first experimental setup is shown in Fig. 5. It consists of a gas chamber with a $\mathrm{CaF}_{2}$ window, inside which any of the mentioned above detectors can be installed, a monochromator combined with a Hg lamp and a gas system allowing to pump the chamber or flush it with various gases: $\mathrm{Ne}, \mathrm{Ar}$ or mixtures of $\mathrm{Ne}$ with $\mathrm{CH}_{4}$.

In all tests we used detectors for which cathodes were coated with CsI layers ( $0.35 \mu \mathrm{m}$ thick).

In this setup we could measure either the photocurrent from various electrodes, in order to evaluate the gas gain and the photoelectron collection efficiency or the charge signals produced by the avalanches.

The procedure followed to measure the quantum efficiency measurements is described in [14], [15].

The second experimental set up is shown in Fig. 6. It consists of a specially designed gas chamber allowing the cooling to cryogenic temperature to take place as well as being pumped or flushed with various gases. Inside the gas chamber a single or double (operating in a cascade mode) hole-type detector can be installed.

In the case of low temperature tests the detector was placed inside the dewar filled with liquid nitrogen $(78 \mathrm{~K})$ or a mixture of dry ice with alcohol $(\sim 195 \mathrm{~K})$ or alcohol with $\mathrm{LN}_{2}(\sim 165 \mathrm{~K})$.

\section{RESULTS}

The use of strip-type spark-protective GEMs can be attractive in many applications. As an example, we will present in this paper the results of some studies oriented to their application to RICH and dark-matter noble liquid detectors.

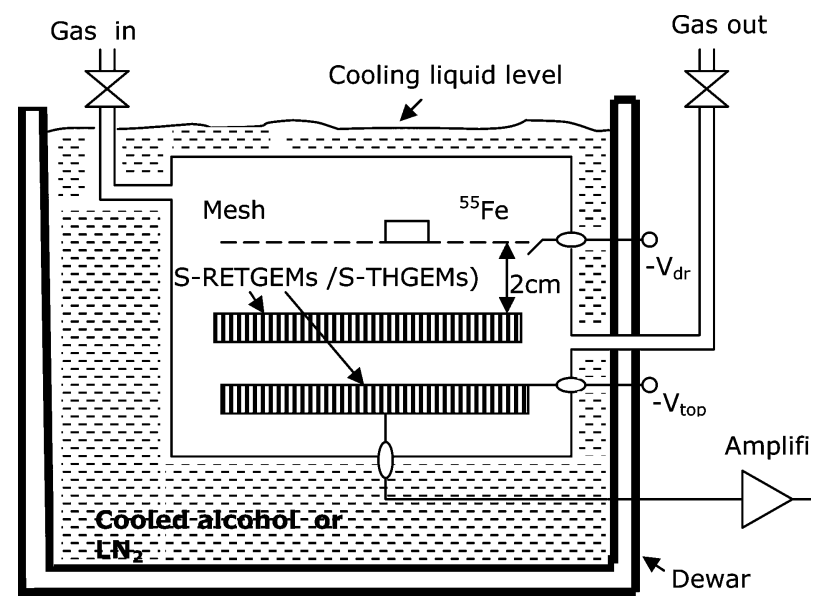

Fig. 6. A schematic drawing of the experimental setup used for the tests at cryogenic temperatures.

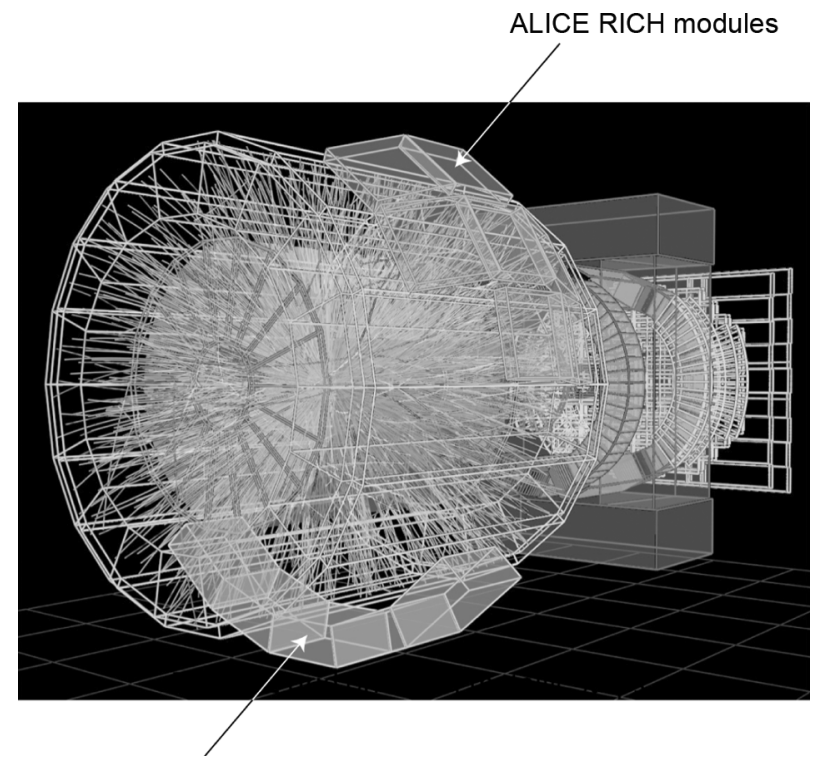

Possible position of VHMPID modules

Fig. 7. A view of the ALICE detector showing the present position of the RICH detectors (right top) and a possible location of the VHMPID modules (in the presently empty space not occupied by any other detector). Simulated tracks are also shown superimposed inside the whole detector (from [16]). Because VHMPID modules are far away from the collision point the flux of charged particles in the region of the VHMPID is rather low and this allows using holetype gaseous multipliers with resistive electrodes or resistive strips.

\section{A. Test Oriented to RICH Applications}

In the framework of the ALICE experiment at LHC, it has been proposed to build a Very High Momentum Particle Identification Detector (VHMPID) with the aim to upgrade the current ALICE layout [16]. The VHMPID should be able to identify, on a track-by- track basis, protons up to $26 \mathrm{GeV} / \mathrm{c}$ enabling to study the leading particles composition in jets (correlated with the $\pi^{0}$ and/or $\gamma$ energies deposited in the electromagnetic calorimeter).

Due to the very limited free space available now in the ALICE detector, the VHMPID will be composed by several small $\left(\sim 1 \times 1 \times 1 \mathrm{~m}^{3}\right)$ modules (see Fig. 7).

The design of the VHMPID module which is presently under study is shown in Fig. 8. It will be a focusing-type RICH de- 


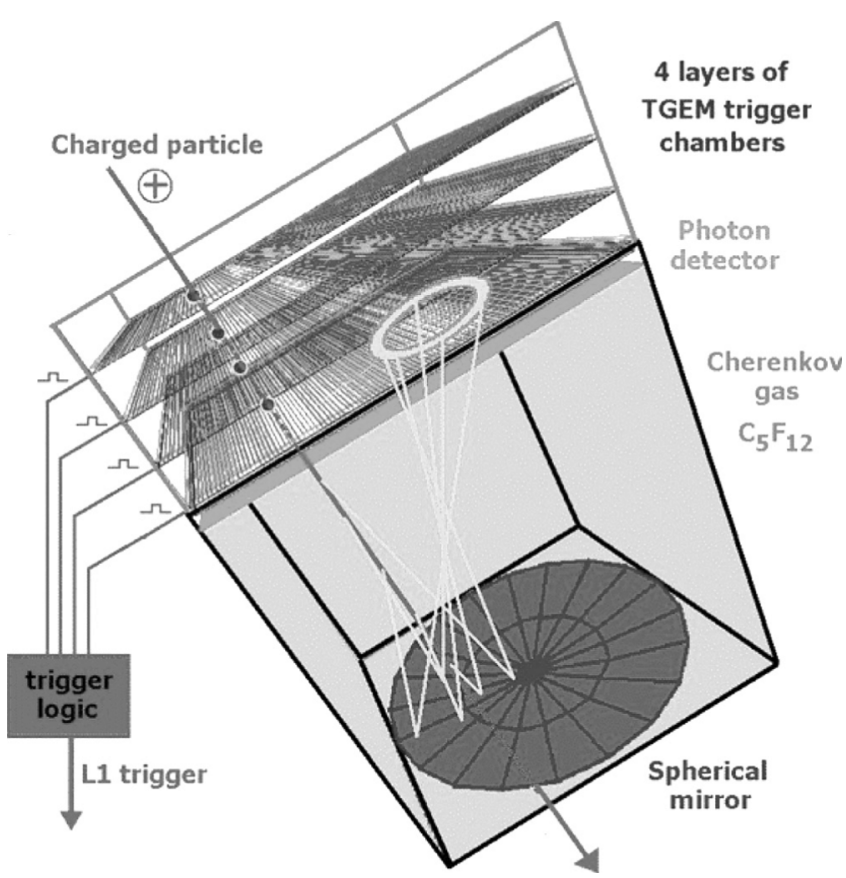

Fig. 8. A schematic drawing of the VHMPID module.

tector with a gaseous radiator $\left(\mathrm{CF}_{4}\right.$ or $\left.\mathrm{C}_{5} \mathrm{~F}_{12}\right)$. The key element of the VHMPID design is a compact planar photodetector. Simulations show that it should have a few $\mathrm{mm}$ position resolution and at least $12 \%$ quantum efficiency at $185 \mathrm{~nm}$ [17]. Because the main task of the VHMPID photodetector will be to detect single photoelectrons produced by Cherenkov radiation, it should operate at a gas gain above $10^{5}$ and thus it will have an elevated risk of sparking. This is why an efficient spark protection of the photodetector is absolutely necessary. Therefore, one of the attractive candidates for the VHMPID photodetector could be a CsI coated hole-type structure protected either with a resistive layer or with in-situ resistors.

In order to choose the most suitable detector for the VHMPID, we performed comparative studies of hole-type detectors described in paragraph II using the setup shown in Fig. 5. In particular, we have measured their maximum achievable gas gains, quantum efficiency and photoelectron collection efficiency.

Fig. 9 shows the gain versus voltage curves measured with a S-RETGEM for two polarities of the electric field in the drift region: a negative one $\mathrm{E}_{\mathrm{dr}}=-250 \mathrm{~V} / \mathrm{cm}$ and a positive one (inversed polarity) $E_{\mathrm{dr}}=+250 \mathrm{~V} / \mathrm{cm}$ [11]. The inversion of the electric field in the drift region allows to suppress the contribution of the natural radioactivity and to additionally increase the maximum achievable gains (see the introduction and (1). Note that the reversed-field method has already been successfully implemented in cascaded-GEM photon detectors with reflective CsI photocathodes, to suppress charged-particle background in high energy physics experiments [18], [19].

From Fig. 9 one can see that at every polarity of the electric field in the drift region, the gas gain achieved in $\mathrm{Ne}$ is an order of magnitude higher than in Ar-based gases. Note that in Ar-based mixtures the operational voltages were considerably

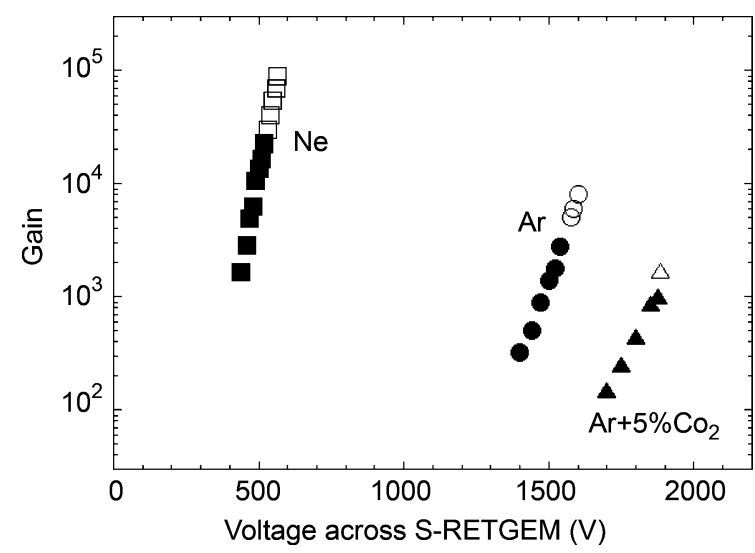

Fig. 9. Gains versus voltage curves for S-RETGEM measured with UV light in $\mathrm{Ne}, \mathrm{Ar}$ and $\mathrm{Ar}+5 \% \mathrm{CO}_{2}$. Open symbols-results obtained with reversed drift field.

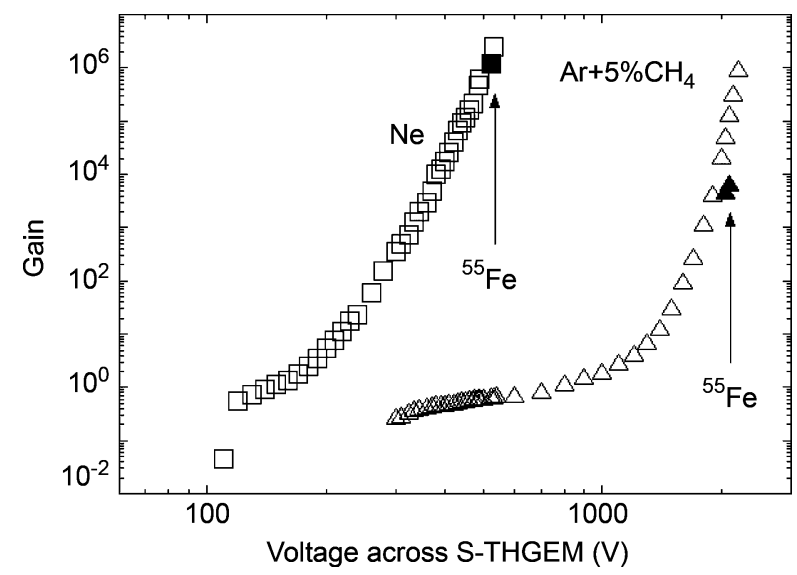

Fig. 10. Gains versus voltage curves for S-THGEM measured with UV light in $\mathrm{Ne}$ and $\mathrm{Ar}+5 \% \mathrm{CH}_{4}$. Filled symbols indicate the maximum gains achieved with an ${ }^{55} \mathrm{Fe}$ source. Note that very similar results were earlier obtained with usual THGEM [20].

higher that in Ne. Thus we can conclude that probably breakdowns in the Ar-based mixtures are mainly triggered by the hole imperfections while in Ne-filled detector the Raether limit could be reached.

Fig. 10 shows gain versus voltage curves measured with a S-THGEM. One can see that in the case of the detection of the UV light, the detector can operate at gains up to $10^{6}$. We attribute these much higher achievable gain than in the case of S-RETGEM to better quality of production of THGEM compared to the screen printed S-RETGEM. Indeed, it was already observed in [10] that the maximum achievable gains of the RETGEMs manufactured by the screen printing technology are lower than gains achieved with RETGEMs whose electrodes were made of resistive Kapton. One also can see that in Ar the maximum gains achieved in the presence of ${ }^{55} \mathrm{Fe}$ source were only $\sim 10^{4}$, which is consistent with the Raether limit for this gas (see formula 1).

Gains achieved with S-GEMs are shown in Fig. 11. As one can see, the maximum sustainable gains of our present S-GEM prototypes were relatively low, typically a few hundred. This can also be attributed to the low production quality of the S-GEMs. 


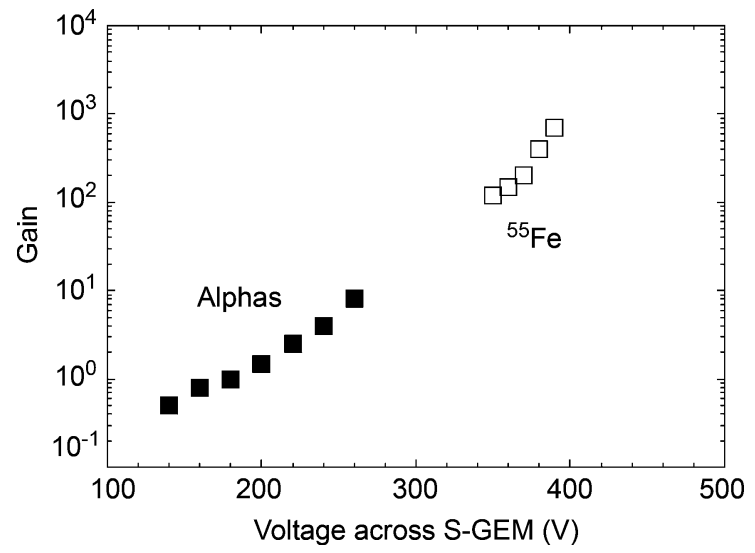

Fig. 11. Gains versus voltage curves for S-GEM measured in Ar with alphas and ${ }^{55} \mathrm{Fe}$.

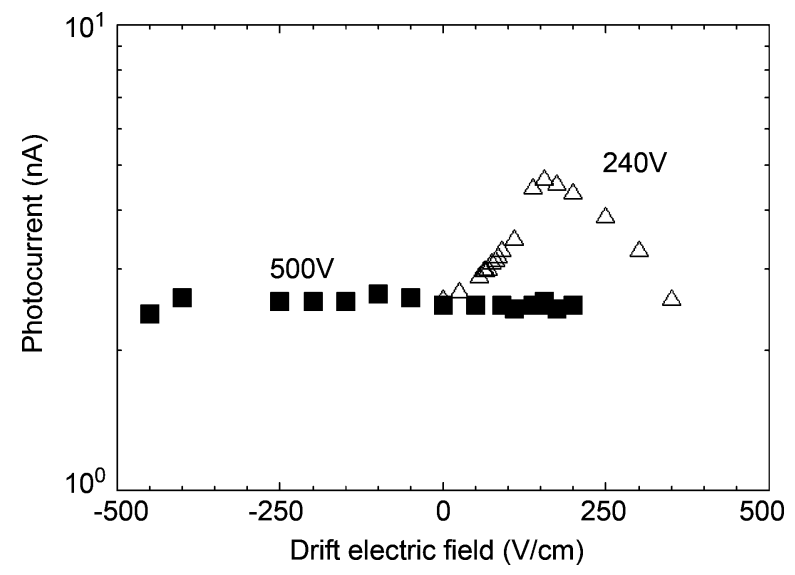

Fig. 12. Photocurrent versus drift field measured with S-THGEM in Ne. Numbers near the curves indicate the voltages applied across the S-THGEM.

However, one should take into account that in general the maximum achievable gain of GEMs is usually $\sim 10$ times lower than those of THGEMs or Kapton RETGEMs [12]. Thus it looks that S-RETGEM and S-THGEM will be more appropriate detectors for VHMPID.

Since it was observed that the polarity of the drift voltage $V_{\mathrm{dr}}$ may affect the maximum achievable gain of some detectors especially when operating in $\mathrm{Ne}$ (see for example Fig. 9), we performed measurements of the signal amplitudes from the anode of the strip hole-type detectors (at the given voltage across the detector $\mathrm{V}_{\mathrm{det}}$ ) as a function of the $\mathrm{V}_{\mathrm{dr}}$. The obtained results were as follows.

Typically, at low values of the $V_{\text {det }}$ the anode signal sharply depended on $\mathrm{V}_{\mathrm{dr}}$ (as an example, see the curve with triangles shown in Fig. 12), however with the increase of the $V_{\text {det }}$ the dependence on the drift voltage became weaker and weaker (see curves with square symbols presented in Figs. 12 and 13). Thus at high gas gains the shape of the gain versus voltage curves were not sensitive anymore to the drift voltage probably indicating that due to the increasing contribution of a dipole-type electric filed created near the holes, all photoelectrons extracted

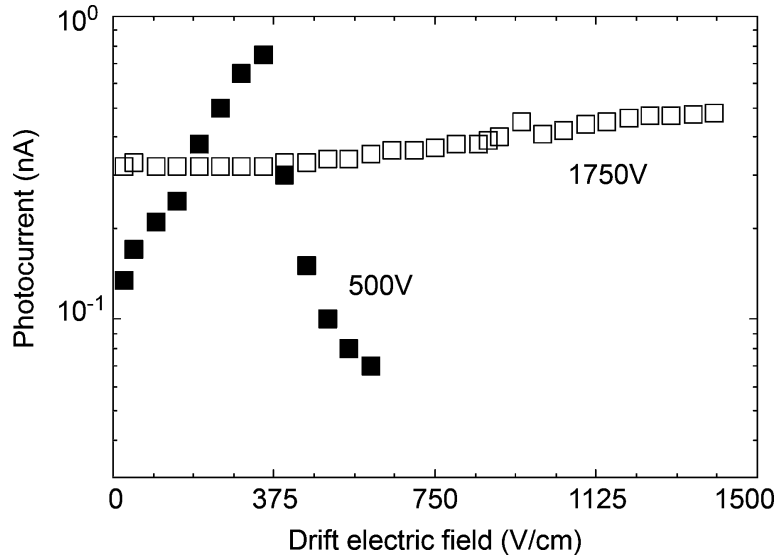

Fig. 13. Photocurrent versus drift field measured with S-THGEM in Ar + $5 \% \mathrm{CH}_{4}$. As in the previous figure, the numbers near the curves indicate the voltages applied across the S-THGEM.

from the CsI photocathode were drifted to the holes, efficiently penetrated into the holes (independently of the $\mathrm{V}_{\mathrm{dr}}$ ), multiplied there and finally collected an the anode electrodes. Of course, this dipole field (and consequently the photoelectron collection efficiency to the S-THGEM holes) depends on the detector geometry: its thickness, diameter of holes, their pitch and the strips width. For example, in the case of the 0.4 thick THGEM a much sharper dependence on $\mathrm{V}_{\mathrm{dr}}$ was observed [13].

To confirm our results, in the next set of experiments, we measured the photoelectron collection efficiency and quantum efficiency of S-RETGEM and S-THGEM.

Results obtained with both of these detectors were identical so for simplicity we will present below only the experimental data for the S-THGEM. Fig. 14 shows photocurrents (produced by a Hg lamp) measured on the drift's electrode (see Fig. 5) as a function of the $V_{\mathrm{dr}}$ under conditions when the chamber was pumped or filled with various gases. In the case of vacuum, the photocurrent reached saturated value $I_{v a c}$ at $V_{\mathrm{dr}} \geq 100 \mathrm{~V}$. In the gas however, due to the well known back diffusion effect [22], the photocurrent rather slowly increased with the $\mathrm{V}_{\mathrm{dr}}$. For example, in the case of $\mathrm{Ne}$, the photocurrent reached $\sim 50 \%$ of the vacuum level at $\mathrm{V}_{\mathrm{dr}}=800 \mathrm{~V}$ indicating that only $50 \%$ of photoelectrons were extracted from the CsI photocathode at this particular voltage. In mixtures of $\mathrm{Ne}$ with $\mathrm{CH}_{4}$ the extraction efficiency was higher: $\sim 60 \%$ for $\mathrm{Ne}+5 \% \mathrm{CH}_{4}$ and $\sim 70 \%$ for $\mathrm{Ne}+25 \% \mathrm{CH}_{4}$ at $\mathrm{V}_{\mathrm{dr}}=1 \mathrm{kV}$.

The quantum efficiency of the T-GEM can be defined as:

$$
\mathrm{Q}(\lambda)=\mathrm{Q}_{\mathrm{vac}}(\lambda) \mathrm{k}\left(\mathrm{V}_{\mathrm{dr}}, \mathrm{V}_{\mathrm{det}}\right) \varepsilon\left(\mathrm{V}_{\mathrm{dr}}, \mathrm{V}_{\mathrm{det}}\right)
$$

where $\mathrm{Q}_{\mathrm{vac}}$ is the quantum efficiency of its CsI photocathode measured in the vacuum at a wavelength $\lambda, \mathrm{k}$ is the extraction efficiency in the gas and $\varepsilon$ is the collection efficiency of the extracted photoelectrons in detector's holes.

The $\mathrm{Q}_{\mathrm{vac}}$ was evaluated with respect to the quantum efficiency of the TMAE vapors $\mathrm{QTMAE}_{\mathrm{TM}}(\lambda)$ as it was already done in 


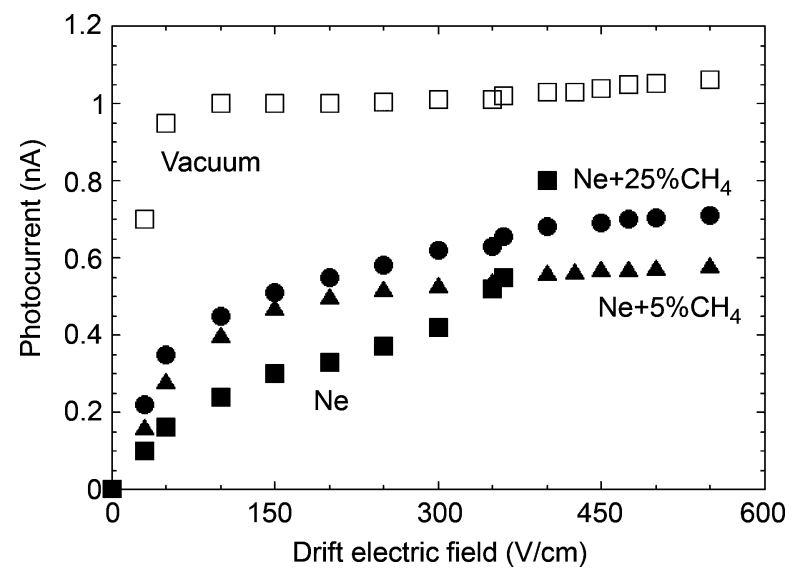

Fig. 14. Photocurrent versus drift field measured in vacuum, $\mathrm{Ne}, \mathrm{Ne}+5 \% \mathrm{CH}_{4}$ and $\mathrm{Ne}+25 \% \mathrm{CH}_{4}$. The sharp current rise at $\mathrm{V}_{\mathrm{dr}}>650 \mathrm{~V}$ in $\mathrm{Ne}$ is due to the electroluminescence effect. These results confirm measurements reported in [21].

[15]. For the same geometrical arrangement of the light source and the detectors:

$$
\mathrm{Q}_{\mathrm{vac}}(\lambda)=\frac{\mathrm{Q}_{\mathrm{TMAE}}(\lambda) \mathrm{I}_{\mathrm{vac}}(\lambda)}{\mathrm{I}_{\mathrm{TMAE}}(\lambda)}
$$

where $\mathrm{I}_{\mathrm{TMAE}}(\lambda)$ is a photocurrent value in the reference TMAE detector. Calculations from formula (3) show that the measured current $I_{\mathrm{vac}}=1 \mathrm{nA}$ (see Fig. 14) corresponds to the $\mathrm{Q}_{\mathrm{vac}}=17.8 \%$ at $185 \mathrm{~nm}$ and respectively photocurrent values: $0.5 \mathrm{nA}, 0.6 \mathrm{nA}$ and $0.7 \mathrm{nA}-$ measured in $\mathrm{Ne}, \mathrm{Ne}+5 \% \mathrm{CH}_{4}$ and $\mathrm{Ne}+15 \% \mathrm{CH}_{4}$ correspond to quantum efficiency values of $8.9 \%, 10.7 \%$ and, $12.4 \%$.

Because $\mathrm{Q}$ depends on $\mathrm{V}_{\mathrm{dr}}$ and $\mathrm{V}_{\mathrm{det}}$, we also performed quantum efficiency measurements at high gas gains (in this case in counting mode -see [14] for details). The results obtained at $\lambda=185 \mathrm{~nm}$ in $\mathrm{Ne}, \mathrm{Ne}+5 \% \mathrm{CH}_{4}$ and $\mathrm{Ne}+15 \% \mathrm{CH}_{4}$ were the following: the quantum efficiencies were $12.3 \%, 13.8 \%$ and $14.7 \%$ respectively.

As one can see, the quantum efficiencies measured in the counting mode were typically $20-30 \%$ higher than values obtained form the current measurements. This is probably due to the fact that at high $\mathrm{V}_{\text {det }}$ the electric field on the top detector's electrode may reach values of $\sim 10 \mathrm{kV} / \mathrm{cm}$ [13] and at such high electric field the coefficient $\mathrm{k}$ may approach the value close to unity.

The main conclusion from these studies is that S-THGEMs and S-RETGEMs could be promising candidates for the VHMPID: they can operate at high gas gains and have sufficiently high quantum efficiency.

\section{B. Test Oriented for Dark Matter Detectors}

At present, several groups are considering the use of holetype gaseous multipliers (GEMs, THGEMs) for the detection of the UV light and primary electrons produced by recoils in noble liquid dark matter detectors (see for example [23] and references therein). Most of early studied were focus to demonstrate that with cascaded GEMs operating in cooled noble gases one can

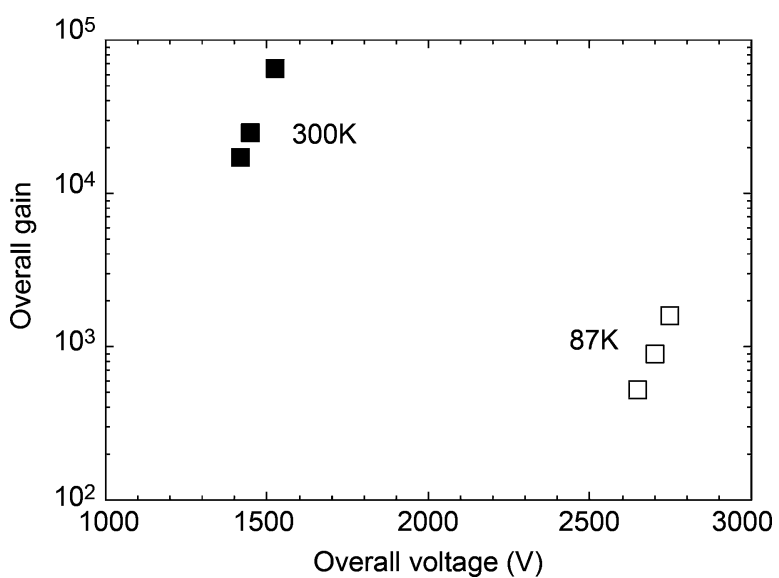

Fig. 15. Gain versus voltage curves measured with double S-THGEM in Ar at $300 \mathrm{~K}$ and $78 \mathrm{~K}$.

detect charges produced by X-rays in these gases or extracted from the noble liquids (see [24] and references therein). The aim of our series of works (see for example [25]-[28] and references therein) was to investigate if hole-type gaseous multipliers coated with CsI layer also operate stably at cryogenic temperatures and can be used for the detection of scintillation light. It was demonstrated that CsI coated GEMs, capillary plates as well as THGEMs have high quantum efficiencies at cryogenic temperatures for the UV photons and thus can replace expensive photomultipliers usually used in cryogenic TPCs for the detection of the scintillation light.

Recently the Novosibirsk group confirmed our results obtained with CsI coated GEMs [29] and also confirmed that a THGEM is a very robust detector capable of operating stably at cryogenic temperatures up to $78 \mathrm{~K}$ [23]. This group also investigated the operation of the old version of the screen-printed resistive GEM (described in [10]) and fully confirmed our results obtained with this detector at room temperature [23]. However, in their experiments at $\sim 84 \mathrm{~K}$ the detector failed to operate properly presumably du to the LAr condensation within the resistive GEM holes. Indeed, under the warming-up conditions, when LAr evaporated from the holes, the detector begun operate with some gas gain.

Our own studies of this old design of the screen-printed resistive GEM additionally revel that in $\mathrm{Ne}$ at $77 \mathrm{~K}$ some charging up effect appears.

Because the detectors described in this paper have improved designs (strips instead of conventional unsegmented electrodes so the avalanche charge is collected near the hole where the avalanche happened) or in some cases were made of a different material (resistive Kapton instead of screen-printed coating), their behavior at low temperatures is expected to be more stable. Our preliminary measurements performed with the setup shown in Fig. 6, fully support this assumption. Figs. 15 and 16 show gain curves measured for S-THGEM at $300 \mathrm{~K}$ and $88 \mathrm{~K}$ and for S-RETGEM in Ne and Ar at $300 \mathrm{~K}, 165 \mathrm{~K}$ and 78 K. In Fig. 17 results are presented of the stability measurements at these temperatures. It is evident that the S-RETGEM does not exhibit any strong charging up effect. Similar stability was observed with the S-THGEM. 


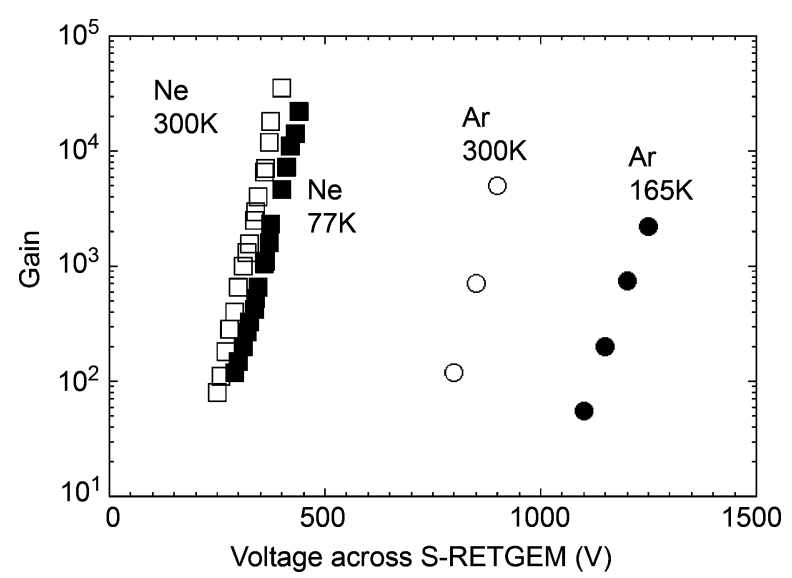

Fig. 16. Gains versus voltage curves for single S-RETGEM measured in Ne at 300 and $78 \mathrm{~K}$ and in Ar at $300 \mathrm{~K}$ and $165 \mathrm{~K}$.

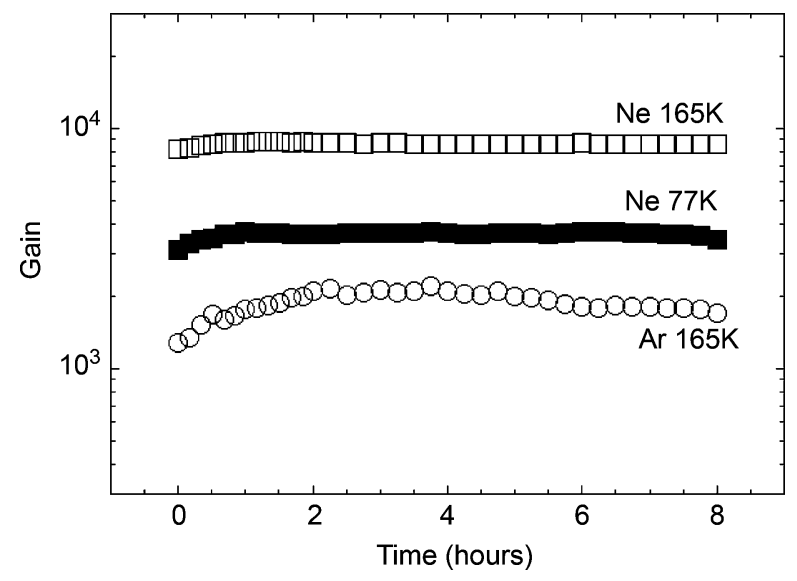

Fig. 17. Gain versus time measured with S-RETGEM at various temperatures in $\mathrm{Ne}$ and Ar.

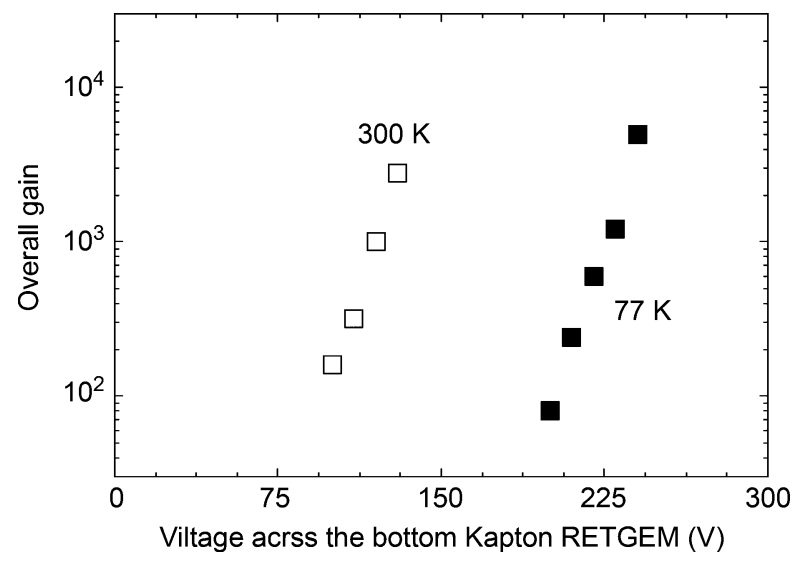

Fig. 18. Gain versus voltage $V_{b}$ across the bottom S-RETGEM. The voltage on the resistor divider which fed the drift electrode and a top S-RETGEM was kept $\mathrm{V}_{\text {total }}=3 \mathrm{~V}_{\mathrm{b}}$.

A brief test was done with the Kapton RETGEM; some results of gain measurements are presented in Fig. 18. In contrast to the screen printed RETGEM (tested in [23]) it also exhibited a rather stable operation-see Fig. 19.

Thus preliminary measurements indicate that all these detector designs could be attractive for noble liquid dark matter detectors.

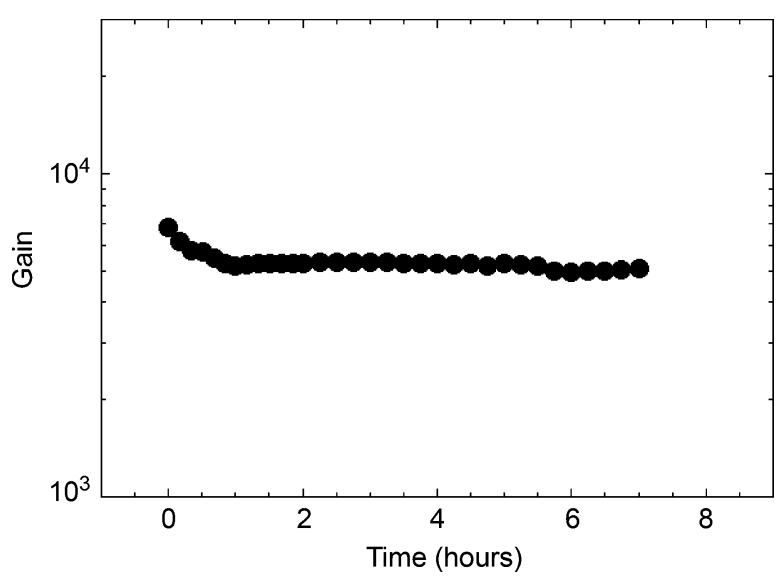

Fig. 19. Gain versus time measured with double Kapton RETGEM in Ne at 77 K.

\section{CONCLUSIONS}

Hole-type detectors with strip electrodes have several advantages over conventional designs:

1) Due to the low strip capacitance, the energy released in the sparks is lower than in traditional designs of GEM-like detectors (with unsegmented electrodes). Resistive layers (or in-situ resistors) allow additional strong suppression of the spark's energy to take place.

2) The strip design offers the possibility of taking position information directly from the strips [11], which could be a convenient option in some applications.

3) In the case of localized defects which triggers breakdowns, the corresponding strip can be disconnected so the detector may remains in an operational condition.

4) Because the strip hole-type detectors can reliably operate at high gas gains and have sufficiently high quantum efficiencies, they can be attractive candidates for the ALICE VHMPID.

5) Preliminary measurements indicate that strip hole-type detectors allow rather stable operation at cryogenic temperatures and thus also can be useful in such applications as dark matter detectors or noble liquid TPCs.

This is why we believe that strip hole-type detectors may have a great future.

\section{ACKNOWLEDGMENT}

The authors would like to thank J. Van Beelen and M. Van Stenis for the technical support throughout this work.

\section{REFERENCES}

[1] A. Del Guerra et al., "High resistance lead glass tubing for RICH counters and electromagnetic calorimeters," Nucl. Instrum. Methods Phys. Res. A, vol. A257, pp. 609-613, 1987.

[2] H. Sakurai et al., "A new proportional counter using a capillary plate," Nucl. Instrum. Methods Phys. Res. A, vol. A374, pp. 341-344, 1996.

[3] F. Sauli, "GEM: a new concept for electron multiplication in gas detectors," Nucl. Instrum. Methods Phys. Res. A, vol. A386, pp. 531-534, 1997. 
[4] L. Periale et al., "Detection of the primary scintillation light from dense $\mathrm{Ar}, \mathrm{Kr}$ and Xe with novel photosensitive gaseous detectors," Nucl. Instrum. Methods Phys. Res. A, vol. A478, pp. 377-383, 2002.

[5] H. Raether, Electron Avalanches and Breakdown in Gases. London, U.K.: Butterworth, 1964.

[6] V. Peskov et al., "The study and optimization of new micropattern gaseous detectors for high -rate applications," IEEE Trans. Nucl. Sci., vol. 48, no. 4, pp. 1070-1074, Aug. 2001.

[7] P. Fonte, V. Peskov, and B. Ramsey, "A study of breakdown limit in microstrip gas counters with preamplification structures," Nucl. Instrum. Methods Phys. Res. A, vol. A416, pp. 23-31, 1998.

[8] W. Amderson et al., "Construction, commissioning and performance of a hadron blind detector for the Phenix experiment at RHIC," in Proc. Nuclear Science Symp., Honolulu, HI, 2007.

[9] R. Oliviera et al., "First tests of thick GEM with electrodes made of resistive kapton," Nucl. Instrum. Methods Phys. Res. A, vol. A576, pp. 362-366, 2007.

[10] G. Agocs et al., "Developments and the preliminary tests of resistive GEMs manufactured by a screen printing technology," J. Inst., vol. 3 P02012, pp. 1-10, 2008.

[11] V. Peskov et al., "Progress in the Development of Photosensitive GEMs With Resistive Electrodes Manufactured by a Screen Printing Technology, Preprint arXiv:0807.2718," pp. 1-9, 2008.

[12] J. Ostling et al., "Study of hole-type gas multiplication structures for portal imaging and other high count rate applications," IEEE Trans. Nucl. Sci., vol. 50, no. 4, pp. 809-819, Aug. 2003.

[13] C. Shalem et al., "Advances in thick GEM-like gaseous electron multiplier-Part I: Atmospheric pressure operation," Nucl. Instrum. Methods Phys. Res. A, vol. A558, pp. 475-489, 2006.

[14] A. G. Agocs et al., "Study of GEM-like detectors with resistive electrodes for RICH applications," Nucl. Instrum. Methods Phys. Res. A, vol. A595, pp. 128-130, 2008.

[15] G. Charpak et al., "Development of a new hole-type avalanche detector and the first results of their applications," IEEE Trans. Nucl. Sci., vol. 55, no. 3, pp. 1657-1663, Jun. 2008.

[16] G. Paic, Report at ALICE Physics Forum, Very High Momentum Identification at ALICE- a Possibility, ALICE Physics Forum, 2008 [Online]. Available: http://indico.cern.ch/conferenceDisplay.py?confId=26371
[17] G. Volpe et al., "Gas Cherenkov detectors for high momentum charged particle identification in the ALICE experiment at LHC," Nucl. Instrum. Methods Phys. Res. A, vol. A595, pp. 40-43, 2008.

[18] D. Mormann et al., "Operation principles and properties of the multi-GEM gaseous photomultiplier with reflective photocathode," Nucl. Instrum. Methods Phys. Res. A, vol. A530, pp. 258-274, 2004.

[19] Z. Fraenkel et al., "A hadron blind detector for PHENIX experiment at RHIC," Nucl. Instrum. Methods Phys. Res. A, vol. A546, pp. 466-480, 2005.

[20] A. Breskin et al., "New results on THGEM operation," in Proc. $2 d$ RD51 Collaboration Meeting, Paris, France, Oct. 2008 [Online]. Available: http://indico.cern.ch/conferenceDisplay.py?confId=35172

[21] A. Breskin and V. Peskov, "THGEM gain investigation: UV vs. X-rays and photoelectron extraction," in Proc. Gaseous Detectors Group Seminar, Aug. 2008, CERN.

[22] V. Peskov, "Secondary processes in gas-filled counters-part II," Sov. Phys. Tech. Phys., vol. 22, no. 3, pp. 335-338, 1977.

[23] A. Bondar et al., "Thick GEM versus thin GEM in two-phase argon avalanche detectors," J. Inst. , vol. 3 P07001, pp. 1-20, 2008.

[24] A. Bondar et al., "Two-phase argon and Xe avalanche detector based on gas electron multipliers," Nucl. Instrum. Methods Phys. Res. A, vol. A556, pp. 273-280, 2006.

[25] L. Periale et al., "The development of gaseous detectors with solid photocathodes for low -temperature applications," Nucl. Instrum. Methods Phys. Res. A, vol. A535, pp. 517-522, 2004.

[26] L. Periale et al., "The successful operation of hole-type gaseous detectors at cryogenic temperatures," IEEE Trans. Nucl. Sci., vol. 52, no. 4, pp. 927-931, Aug. 2005.

[27] L. Periale et al., "Photosensitive gaseous detectors for cryogenic temperature applications," Nucl. Instrum. Methods Phys. Res. A, vol. A573, pp. 302-305, 2007.

[28] L. Periale et al., "A study of the operation of especially designed photosensitive gaseous detectors at cryogenic temperatures," Nucl. Instrum. Methods Phys. Res. A, vol. A567, pp. 381-385, 2006.

[29] A. Bondar et al., "Recent results on the properties of two-phase argon avalanche detectors," Nucl. Instrum. Methods Phys. Res. A, vol. A598, pp. 121-125, 2009. 